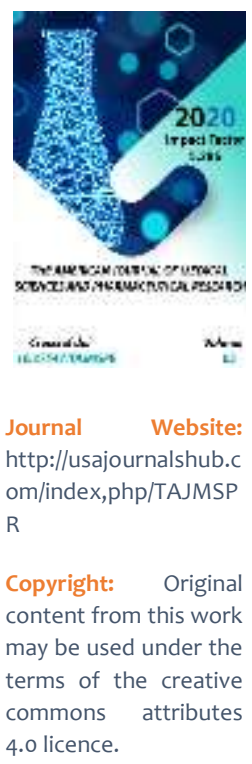

\title{
Prevention Of Anemia In Patients With Tuberculosis
}

Mamatkulova Feruza Xaydarovna

Samarkand State Medical Institute, Teacher Of The Department Of Hematology, Uzbekistan

Mamatova Nargiza Toirjonovna

Samarkand Medical Institute, Lecturer At The Department Of Phthisiology, Uzbekistan

Ruziboeva Oyjamol Narzullaevna

Samarkand Medical Institute, Teacher Of The Department Of Hematology, Uzbekistan

\section{ABSTRACT}

This article include prevention of anemia in patients with tuberculosis. tuberculosis induces a systemic inflammatory state affecting iron homeostasis. patients with tuberculosis often have additional comorbidities such as anemia which can result in poorer treat outcomes. we studied the contribution of anemia and the role of the iron regulatory hormone hepcidin among tuberculosis patients and household contacts.

\section{KEYWORDS}

Tuberculosis, anemia, treatment of tuberculosis.

\section{INTRODUCTION}

Tuberculosis or tuberculosis is an infectious disease of bacterial etiology. The disease is not only medical, but also social: the most susceptible to TB are people with reduced immunity, malnutrition, poor hygiene and poor living conditions. The quality of human life affects the development of the disease. However, the entire population, regardless of age and gender, is at risk of tuberculosis.

The high mortality rate ( 3 million people per year) and the prevalence of the disease are influenced not only by social factors, but also by the long (asymptomatic) period of tuberculosis. This period is the most favorable time for the treatment of tuberculosis and the organism is evaluated by the Mantoux test reaction to determine the presence of infection. 
Many patients with active pulmonary tuberculosis exhibit decreased levels of hemoglobin, which can directly impact tuberculosis -associated morbidity. Anemia can be defined as hemoglobin $(\mathrm{Hb})$ levels below $12.5 \mathrm{~g} / \mathrm{dL}$ for women and $13.5 \mathrm{~g} / \mathrm{dL}$ for men 6 . Anemia can have many causes, including iron deficiency and chronic inflammation. These two distinct mechanisms of anemia present different laboratorial definitions. Anemia caused by iron deficiency is associated with ferreting levels $<30 \mathrm{ng} / \mathrm{mL}$ whereas that caused by chronic disease is linked to ferreting levels $>100 \mathrm{ng} / \mathrm{mL} 7$. In addition, serum concentrations of ferreting are reported to increase in inflammatory conditions such as autoimmune diseases, infections, malignancy and other diseases. In both types of anemia, transferring saturation values drop below $20 \%$. Anemia has been extensively studied in the context of tuberculosis and both iron deficiency-related and chronic diseaseassociated anemia's have been reported. The specific interplay between systemic inflammation, mycobacterium loads in sputum and anemia status in cohort studies is unknown.

As a result of intoxication, changes in the reactivity of the body and tissue damage in patients with tuberculosis, shifts in the hematopoietic system of different nature occur. An essential role is played not so much by the form as by the phase and dynamics of the process. With limited and inactive forms, the number of erythrocytes is usually within the normal range and there are no signs of anemia. With massive infiltrates or cheesy pneumonia, with widespread case us lymphadenitis, specific intestinal lesions, involvement of the liver, spleen, bone marrow in the process, as well as after major pulmonary or postoperative bleeding, a drop in the number of erythrocytes and a change in their morphology (oligo- and polychromasia) are noted, and sometimes megaloblastic, aplastic, or mixed anemia (megaloblastic and iron deficiency). This increases the content of not fully matured erythrocytes - reticulocytes (over $0.5-1 \%$ ) and the number of platelets (more than 250,000-300,000). The erythropoietic function of the bone marrow changes in a similar way. More often and to a greater extent with tuberculosis, but mainly with pronounced, progressive and complicated forms, the leukogram changes. In some cases, there may be moderate leukocytosis (up to 10,000-15,000 leukocytes), less often leukopenia. So, E.L. Kan (1972) observed leukopenia in $20.7 \%$ of patients with limited and easily proceeding forms of the process and in $12.5 \%$ - with destructive and progressive pulmonary tuberculosis. Most often, shifts occur in the leukocyte formula. The number of neutrophils increases both in absolute and in relative terms due to stab forms (up to 15-20\%). At the same time, the content of lymphocytes decreases (up to 8$15 \%$ ). With a pronounced and prolonged infiltrative process and extensive bronchogenic seeding, pathological granularity of neutrophils appears. With dissemination, according to the observations of NN Bobrov (1950) and NA Shmelev (1959), monocytosis increases (up to 10-18\%). The content of eosinophils in the peripheral blood also fluctuates depending on the phase of the process and the allergic state of the body. Their number decreases up to aneosinophilia in severe and prolonged outbreaks of the disease and, conversely, increases (up to 8-10\%) with resorption of infiltrates and pleural effusion, as well as in early forms of primary tuberculosis. In the latter case, monocytosis is often determined simultaneously. But at the same time, there is no pronounced eosinophilia characteristic of "flying" eosinophilic pneumonia of allergic or viral origin, when 
eosinophilic granulomas develop in various internal organs.

Noteworthy is the indication of an increase in the absolute number of basophils with an active tuberculosis process. The platelet profile of peripheral blood is characterized by an increase in the proportion of forms of irritation and degenerative elements, which is due to the degree of activity of the process and intoxication. Thus, the individual phases of pulmonary tuberculosis are characterized by the following hematological syndromes. Infiltration phase: moderate or significant leukocytosis, pronounced shift of peitrophils to the left, normal or increased content of neutrophils with pathological granularity, moderate or significant acceleration of ROE, displacement of the thrombocytogram to the right due to an increase in the content of forms of irritation and degenerative elements. Decay phase: small leukocytosis or normal leukocyte count, an increase in the percentage of neutrophils with pathological granularity and a shift to the left, sometimes with the appearance of myelocytes, a decrease in the number of eosinophils and lymphocytes, a significant acceleration of ROE. Phase of hematogenous dissemination: normal leukocyte count, absolute and relative monocytosis. Resorption phase: an increase in the number of eosinophils and lymphocytes, the absence of a nuclear shift to the left. Compaction phase: normal hemogram indices, sometimes lymphocytosis. The hemogram in patients with tuberculosis changes during treatment. In favorable cases, the quantitative and qualitative composition of blood cells is normalized. Such positive shifts usually occur 23 months later, after a longer period of time after the disappearance of fever and other symptoms of intoxication. Hematological changes disappear the faster, the more successful the therapeutic intervention, that is, the sooner the intoxication stops, the foci and zones of infiltration dissolve, the cavities close, the bacillic excretion stops. At the same time, one should bear in mind the effect on hematopoiesis of various antibacterial drugs. They often cause eosinophilia, in some cases leukocytosis, and more often leukopenia; sometimes at the same time the formation of neutrophils is inhibited up to agrapulocytosis and the lymphoid-reticular reaction can be stimulated. These changes occur as a result of the allergenic or toxic effect of drugs on the bone marrow. With the development of agranulocytosis, it is necessary to stop taking the medication that caused it. Allergy to the drug is determined by laboratory tests (Shelley test, Wanier reaction). The appointment of sodium nucleinate or pentoxil, corticosteroid hormones, multivitamins, blood transfusion helps to restore bone marrow hematopoiesis. Thus, systematic hematological control and the correct analysis of the results obtained are essential for assessing the patient's clinical condition, the dynamics of the process and the effectiveness of the treatment used. Hematological data acquire a certain value in the differential diagnosis of tuberculosis and other respiratory diseases.

\section{REFERENCES}

1. Faris P.M. The effects of recombinant human erythropoietin on perioperative transfusion requirements in patients having a major orthopedic operation / P.M. Faris,M.A. Ritter, R.I. Abels; The American Erythropoietin Study Group // J Bone Joint Surg Am. - 1996

2. Kumar A. Perioperative management of anemia: Limits of blood transfusion and alternatives to it / A. Kumar // Cleveland Clinic Journal of Medicine. - 2009.

3. Laupacis A. Erythropoietin to minimize perioperative blood transfusion: a 
systematic review of randomized trials.

The International Study of Preoperative Transfusion (ISPOT) Investigators / - 1998.

4. The Prevalence and Evolution of Anemia Associated wich Tuberculosis / W. Lee J. Korean. Med. Sci. - 2006.

5. Preoperative administration of epoetin alfa to reduce transfusion requirements in elderly patients having primary total hip or knee reconstruction / R.A. Lofthouse [etal.] // J South Orthop Assoc. - 2000. 